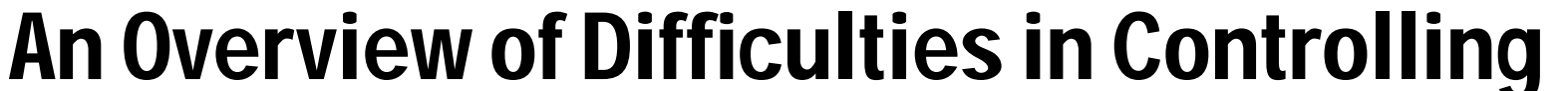

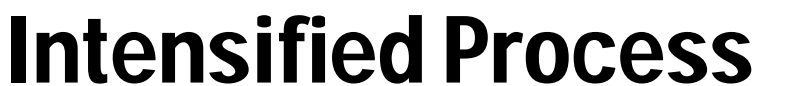

\section{Reza Barzin}

Syamsul Rizal Abd Shukor

Abdul Latif Ahmad

School of Chemical Engineering, Engineering Campus, Universiti Sains Malaysia, 14300 Nibong Tebal, Seberang Perai Selatan, Penang, MALAYSIA

Phone +604-5996402, Fax: +604-5941013

Email: chsyamrizal@eng.usm.my

\begin{abstract}
Process intensification (PI) is currently one of the most significant trends in chemical engineering and process technology. $\mathrm{PI}$ is a strategy of making dramatic reductions in the size of unit operations within chemical plants, in order to achieve production objectives. PI technology is able to change dramatically the whole chemical engineering industry pathway to a faster, cleaner and safer industry. Nonetheless, PI technology will be handicapped if such system is not properly controlled. There are some foreseeable problems in order to control such processes for instance, dynamic interaction between components that make up a control loop, response time of the instrumentations, availability of proper sensor and etc. This paper offers an overview and discussion on identifying potential problems of controlling intensified systems.
\end{abstract}

Keywords: fast processes, hybrid systems, miniaturized devices, process control, process intensification

\section{INTRODUCTION}

As it is widely understood, $\mathrm{PI}$ comprises compact novel equipments, advanced processing techniques and process development methods which compared to conventional ones offer substantial improvement in chemical manufacturing and processing [Stankiewicz 2004]. PI is a strategy of making dramatic reductions in the size of unit operations within chemical plants, whilst maintaining the given production objectives. In recent years, numerous investigations have been done on PI technology that have made huge advancements in making feasible PI concepts and ideas as well as in attempting to establish key design parameters for various process units.

Generally, PI can be divided into two main categories: intensification that has been done by means of instruments, such as micro reactors; 
and, intensification that has been done by means of methods, such as heat integrated reactors [1] (Stankiewicz 2004). Intensification by means of integration of systems is known as hybrid system while intensification through miniaturization results in low volume devices. There are many advantages using PI technology, for instance $\mathrm{PI}$ through miniaturization can significantly reduce resistance to heat and mass transfer. Consequently, it is possible to achieve the high heat transfer needed for explosive reactions that could notbe addressed using conventional devices and to overcome the mass and heat resistance in high viscosity processes like polymerization. In addition to that PI through integration of several unit operations into one common apparatus has the potential to substantially improve the economics of chemical processes if applied to adequate problems (Adrian, Schoenmakers, and Boll 2004).

The need of improved process control is adamant in order to achieve all these advantages because it has always been recognized that in deciding on the best operating conditions, the problem of control will have a direct effect on how the optimal policy will be realized (Mc Greavy 1983). However, from the control point of view PI can be divided into two different categories: intensification done by miniaturization, in which the equipment sizes are reduced whilst maintaining the same throughput; and, intensification do ne by integration, where different functions are integrated into one apparatus. This categorization is due to the different control strategies which are required. Unfortunately, few investigations have been reported the operation and control of intensified systems, especially in the case of low volume intensified devices: micro reactor, spinning disc reactor, static mixer reactor, and monolithic reactor. The inevitability of having downsized-equipment in $\mathrm{PI}$ technologies would make process more subject to being out of control (Keller and Bryan 2000).

Intensification, either through integration or miniaturization, has its special problems. For example, in intensification through miniaturization, due to fast responsiveness of the system, the conventional actuators may not be able to cope with the control system. On the other hand, intensification through integration typically causes strong interactions of different process quantities and a loss in degree of freedom (Adrian, Schoenmakers, and Boll 2004). This paper presents examples on control of hybrid systems and low volume devices, difficulties in the control of intensified processes and proposes solutions. Finally, the simulation results for control of the low volume intensified system are presented.

\section{CONTROL OF HYBRID SYSTEMS}

Adrian, Schoenmakers, and Boll (2004) investigated the control of a divided wall column. In order to control the system, model predictive controllers (MPC) in comparison to the control with a single loop proportional-integral controller concept have been investigated. In addition to the successful result that can be obtained by implementing the proportional-integral controller, the authors showed that the model predictive controller considerably improved control behavior in respect to maximum deviations of the controlled variables and the time to reach steady state.

Toledo et al. (2005) studied an autorefrigerated CSTR polymerization reactor connected to a semiflo oded horizontal condenser. In order to analyze the temperature control of the system, different control algorithms were used, such as: classic proportional-integral control, the long-range predictive QGPC (generalized predictive control (GPC), with restrictions using optimization routine; successive quadratic programming (SQP); and the adaptive predictive STQGPC (QGPC coupled to the identification algorithm RLS). Predictive controllers demonstrate more ability to control the system. It should also be noted that polymerization reactors are typically highly nonlinear chemical processes, with several characteristics, which lead to further difficulties in the development of proper modeling and control for such systems. 
Reactive distillation (RD) has probably received the most attention among other intensified systems from the control point of view. Many of these investigations on RD, proportional-integral controllers applied to the system (Wille et al. 2004; Al-Arfaj and Luyben 2002a, 200b; Bisowarno, Tian, and Tade 2003). Different proportional-integral structures have been applied, for instance SISO structures are being used (Al-Arfaj and Luyben 2000a) with proportional-integral controllers and they have analyzed dual composition control, or single-end composition control, dual temperature control or single-end temperature control. Al-Arfaj and Luyben (2000) evaluated six alternative control structures for an ideal two-product reactive distillation column. The use of a compositional analyzer in the reactive zone to maintain stoichiometric balance was advocated. In order to have an accurate composition analysis for the reactive distillation tray, advanced composition analyzers should be used. Nonetheless, often the results are not reliable due to the limited information of such system.

Two different solutions have been suggested for this problem. One is to use an alternative controlled variable that can reflect the composition of the reactive part. Al-Arfaj and Luyben, (2002b) who applied temperature control in order to control composition of the reactive part instead of using the expensive composition analyzer, and obtained very good results using simple proportional-integral controller for ethylene glycol reactive distillation column. AlArfaj and Luyben (2000) also suggested that the state estimator could be a suitable alternative to the expensive and often unreliable advanced composition analyzer because the estimatorbased control usually used in these cases has imperfect and limited information. Olanrewaju and Al-Arfaj (2006) not only demonstrated that using estimator-based controllers, measurement errors can be minimized but also concluded that the estimator-based control system will give a good performance when an accurate process model of a system is obtained.
In the control of reactive extrusion, high nonlinearity of the process and measurement noise and large time delay (dead time) are major control problems. Curry et al. (1988) developed a control scheme to control reactive extrusion that is used in polypropylene degradation using a proportional-integral controller. High nonlinearity and large dead time of the extrusion process introduce relatively large error to the system. In order to compensate for this problem a controller scheme is developed (Pabedinskas, Cluett, and Ballic 1989) to control the amount of degradation. Thus, to improve the performance of the digital proportional-integral controller, gain scheduling and Smith predictor were used in conjunction with the proportional-integral controller in order to compensate for the nonlinear process gain and process delay. The best result was obtained from using proportional-integral with Smith predictor. Pabedinskas and Cluett (1994) designed and analyzed a process control strategy for reactive extrusion polypropylene degradation process that also produced very good results for load disturbance. An empirical model of the reactive degradation process has been used to describe the process dynamic and disturbance characteristic. Comparing simulation results, pole placement $(P P)$ is preferred to minimum variance $(M V)$, constrained minimum variance (CMV), and applied to the actual reactive extrusion. The PP controller has successfully addressed the control objectives.

\section{CONTROL OF LOW VOLUME DEVICES}

Little investigation has been reported on low volume devices. Wille et al. (2004) studied the application of microdevices in combination with conventional devices. In the study, continuous coupling of the yellow and red model pigments in several microdevices under various aspects have been investigated. In the system, controlling of exact dosing of the reactants using two dosing pump have been studied. In order to control the system, a set of PID controller was applied to the system and the results have been 
analyzed. For processes based on microreactors, results obtained from the investigation give much motivation for further improvement of an automated control, while using conventional and commercially available digital measurement as well as control equipment.

Palusinski etal. (2001) raised the problem of inapplicability of digital processing for controlling fast systems and suggested analog control. The author proposed field programmable analog arrays in order to program the controllers for different process states. Different process models can be stored in FPAA to be applied whenever is necessary.

\section{DIFFICULTIES IN CONTROL OF INTENSIFIED PROCESS}

Due to the complexity and fast responsiveness of intensified processes, controlling of PI operation units usually faces some difficulties. However, this problem seems to be different for each category of intensified devices as a result of their different natures. Control of hybrid system faced with a very complex system is usually relatively slow while control of low volume devices faced with very fast processes usually is not complex.

\section{Control problem of hybrid systems}

Integration of several unit operations into one common apparatus in hybrid systems causes complexity in the control of the hybrid systems because integration typically causes strong interactions of different process quantities and a loss in the degree of freedom. This may require a more elaborate automatic control scheme. For the case of hybrid systems, advanced digital control strategies seem to be suitable alternative to the conventional control strategies (Toledo et al. 2005; Adrian, Schoenmakers, and Boll 2004). For instance in MPC the effects of all manipulated variables to all controlled variables are regarded simultaneously. This makes it possible to handle more manipulated variables than controlled variables. And also all manipulated variables react immediately after detecting the disturbance. This immediate reaction results in favorable control in hybrid systems. In the case of reactive distillation, as mentioned earlier, estimator-based control have been applied to the system in order to compensate for the limited and inaccurate information of composition analyzer. Results showed that an improved control behavior can be achieved using estimator-based control (O lanrewaju and Al-Arfaj 2006).

In addition, using advanced controllers can improve the economics of the process. For instance, in the case of divided wall column, this could be shown that they can be operated using conventional control strategies implemented with classical proportional integral controllers. However, due to the rather poor control behavior of classical strategies, some of the expected savings in energy have to be neglected to run the process at a more stable operation point and some of the expected savings in investment can not be realized due to increased safety margins (Adrian, Schoenmakers, and Boll 2004). In spite of the fact that the effort for modeling and tuning and finally starting up the process using MPC is about three times higher than for the proportional-integral control concept, the use of MPC should lead to considerable economical benefits if processes can be operated closer to their capacity limit and near their energetic optimal but less stable operation point. Because such robust and fast control strategies are able to operate near operation limits and not allowing system to go beyond those limits.

\section{Control problem of low volume devices}

In the control of hybrid systems, complexity and instrumentation limitations are the main reasons for inevitable use of advanced digital control strategies. Whereas fast responsiveness of low volume devices is the main problem in the control of intensified systems. Having very short residence time due to the small volume while maintaining almost the same throughput 
as conventional devices results in very fast responsive devices which cause problems:

Instrumentation problems. Due to the fast responsiveness of intensified processes currently available instruments may not be suitable devices to be applied for intensified processes. For example, conventional valves may not be fast enough to do the final control action. On the other hand, currently available measurement devices may be too slow to measure the controlled parameter in order to do suitable control action in conventional feedback strategies.

Micro valves seem to be the suitable devices to use in conjunction with low volume devices because of their fast response - as fast as $2 \mu \mathrm{s}$ for polymer micro valves (Rogge, Rummler, and Schomborg 2004). But because of their small size, only very little flow can pass through them so they cannot be applied for high-throughput miniaturized devices unless a large number is used them in parallel, which seems uneconomical.

Instrumentation error to the system. In conventional process control applications, the dynamic behavior of the final control elements and transducer can safely be neglected in comparison to the dynamics of the process itself as it has deemed that the process has a bigger residence time compared to the instruments. Nevertheless, this is not true for PI system as miniaturization of equipment units inescapably means that the residence times of intensified systems will indefinitely be smaller than conventional units, typically of the order of fractions of a second. Consequently, for such systems, the dynamic behavior of the final control elements and transducer cannot be negligible and they should be considered in the controller design calculations (Abd Shukor and Tham 2004).

Controller tuning relation. Conventional controller setting relation may not be applicable in order to set controller setting for control system of low volume devices. In almost all conventional controller tuning relations, measurement has not been considered in the calculations. Consequently, in order to tune the controller for low volume intensified systems, different controller tuning relations should be developed. This would open new areas in the process control field. A more detailed controller design approach could be adopted in order to make sure every element in the control loop would be taken into account in order to achieve appropriate overall control performance.

Difficulty in using digital control. Digital control techniques are the common and most appropriate for systems requiring slow response, with a high order of complexity and limited requirement for adaptability. Control in low volume devices involve high-frequency signals that cannot be handled by inherently slow digital processing because of its sampling nature Rogge, Rummler, and Schomborg 2004). Hence, analog control should be applied to the system and this poses some difficulties in the case of complex processes. But, as mentioned earlier, field programmable analog arrays could be suitable to overcome this problem.

Difficulties caused by high time delay. Process time delay exists in all systems because material and energy physically moves in the process or plant. This time delay is normally acceptable for conventional systems comparing to the process time constant which is a couple of minutes. But for low volume intensified systems sometimes this time delay is even higher than the process time constant making serious difficulties in their control.

In order to better present a clearer picture of the difficulties in controlling intensified processes, simulation results for low volume intensified systems are discussed in the following sections.

\section{SIMULATION FOR A LOW VOLUME INTENSIFIED SYSTEMS}

Feedback control loop including the measurement part was used in order to do the simulations shown in Figure 1.

$Y_{s p}(s)$ and $Y(s)$ are set-point and process output, respectively. $G_{v}(s)$ and $G_{m}(s)$ are the transfer functions of the valve and measurement parts that are assumed to be first order transfer 


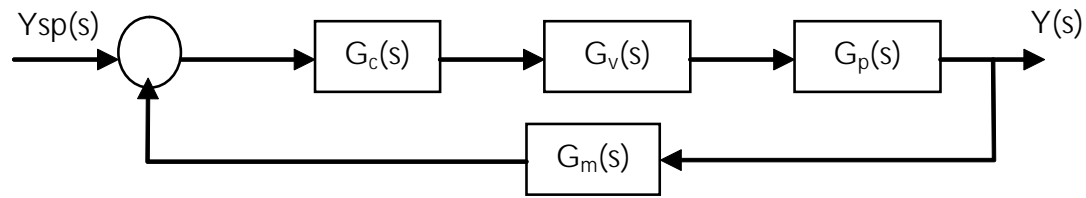

Figure 1. Feedback control loop

function. $G_{p}(s)$ is the process transfer function which is a first order plus time delay. The transfer functions of $G_{v}(s), G_{p}(s)$, and $G_{m}(s)$ are given as:

$$
\begin{aligned}
& G_{V}=\frac{k_{V}}{\tau_{V} s+1} \\
& G_{P}=\frac{k_{P} e^{-\theta s}}{\tau_{P} s+1} \\
& G_{M}=\frac{k_{M}}{\tau_{M} s+1}
\end{aligned}
$$

Controller $\left(G_{c}\right)$ is designed using direct synthesis (DS) which resulted in a PID+D2 controller plus a first order low pass filer as shown in Eq. (4):

$$
G_{C}=K_{C}\left(\tau_{d^{2}} s^{2}+\tau_{d} s+\frac{1}{\tau_{i} s}+1\right) \times G_{K}
$$

The controller components are as follow:

$$
\begin{aligned}
& \tau_{d^{2}}=\frac{\tau_{M} \tau_{P} \tau_{V}}{\left(\tau_{M}+\tau_{P}+\tau_{V}\right)} \\
& \tau_{d}=\frac{\left(\tau_{M} \tau_{V}+\tau_{P} \tau_{V}+\tau_{M} \tau_{P}\right)}{\left(\tau_{M}+\tau_{P}+\tau_{V}\right)} \\
& \tau_{i}=\left(\tau_{M}+\tau_{P}+\tau_{V}\right) \\
& K_{C}=\left(\frac{\left(\tau_{M}+\tau_{P}+\tau_{V}\right)}{\left(\lambda+\tau_{M}+\theta\right)}\right)
\end{aligned}
$$

$$
G_{K}=\frac{1}{\left(\frac{\lambda \tau_{M}}{\left(\lambda+\tau_{M}+\theta\right)} s+1\right)}
$$

Simulations were done for a wide range of values in order to cover almost all possible values for the case of low volume intensified systems. Integral absolute error (IAE) were used for performance analysis.

Process time constant were peged at 1,3 and 10 to mimic fast, medium and slow processes respectively. Figure 2 shows that when the same range of time delay, $\theta$, is applied, overly controlled performance for fast process deteriorates significantly in the range of large time delay. The inability of the system to handle a time delay greater than the process time constant makes the system unstable and, consequently, a large value of IAE. Figure 3 (a) is evidence of the that, for a fast system $\left(\tau_{p}=1\right)$, having a time delay $\theta=3$ makes for a totally unstable process (dotted line). The same goes for a medium system with a time delay higher than the process time constant. The system shows instabilities in which a bigger time delay value increases error in the system.

On the other hand, instrumentation also introduces error to the system when slow measurementmakesthe system sluggish, resulting in higher error as shown in Figure 3(b).

\section{CONCLUSION}

Control of intensified processes, either for hybrid systems or low volume systems, faces many difficulties to which due to characteristics specific to each group, solutions have been proposed. Model-based design methods such as 
(a)

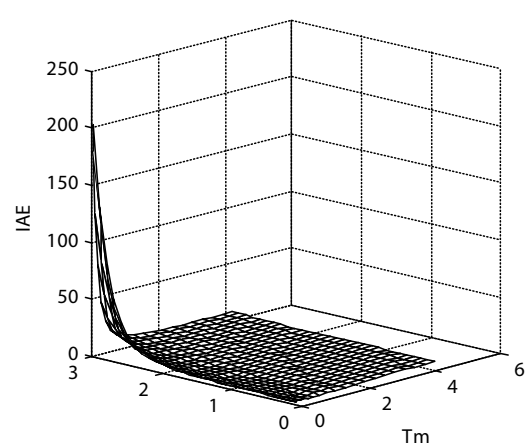

(b)

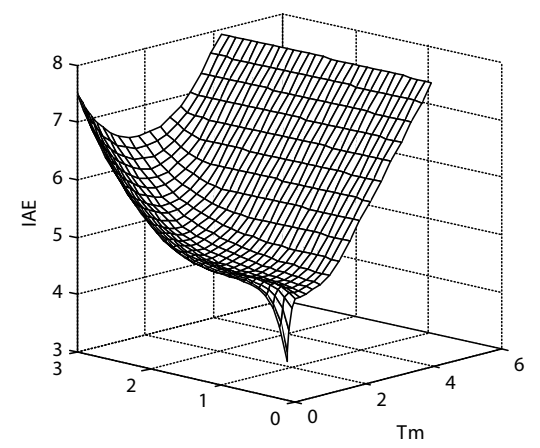

(c)

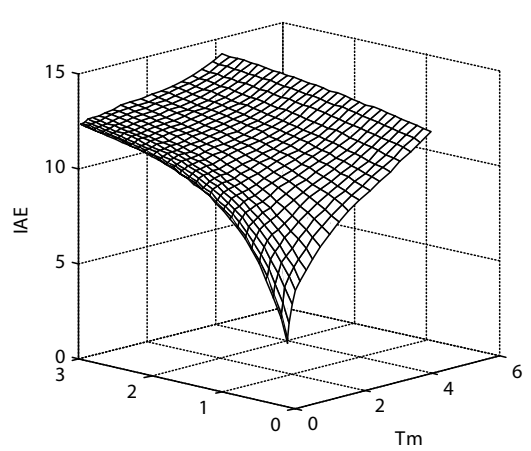

Figure 2. IAE value for (a) $\tau_{p}=1$, (b) $\tau_{p}=3$, and (c) $\tau_{p}=10$ at $\tau_{v}=0.7$

(a)

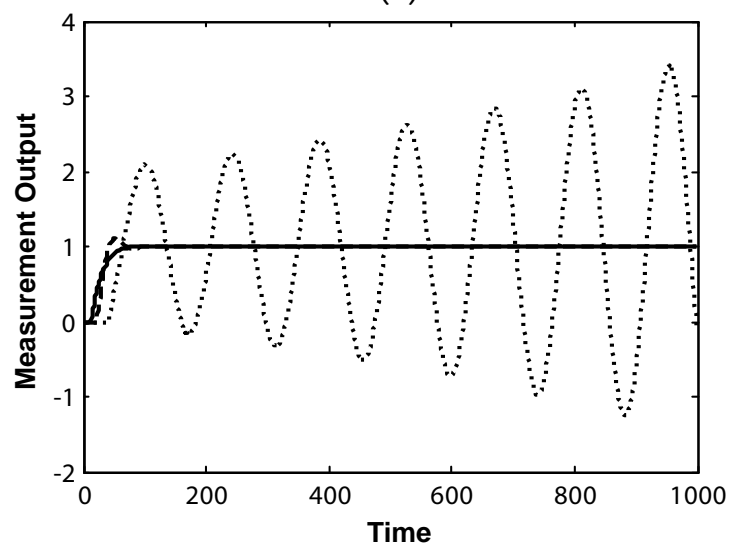

(b)

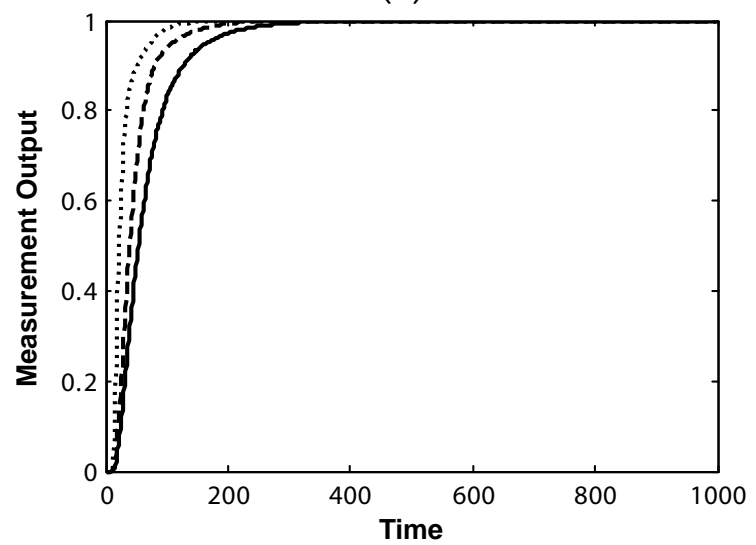

Figure 3. Step response for a fast system with $\tau_{p}=1$ at (a) $\theta=0.1$ (dotted line), $\theta=1$ (dashed line), and $\theta$ $=3$ (solid line); and, (b) at different $\tau_{\mathrm{m}}: \tau_{\mathrm{m}}=0.1$ (dotted line), $\tau_{\mathrm{m}}=2$ (dashed line); and $\tau_{\mathrm{m}}=4$ (solid line)

direct synthesis (DS) or internal model control (IMC), are possible alternatives in order to design a suitable controller as well as controller tuning. Simulation results show that time delay plays a critical role in the control of intensified systems where a time delay that easily can be handled in conventional systems can deteriorate the performance of an intensified system and make system totally unstable. And also measurement should be fast enough to accommodate the fast responding of the system. Thus, control of PI systems should be given more emphasis and further investigations should be done; if not, it is possible that the current developed devices would not be a complete material for commercialization.

\section{ACKNOWLEDGMENT}

The authors gratefully acknowledge Universiti Sains Malaysia for funding this project in the form of a short-term grant. 


\section{REFERENCES}

Abd Shukor, S. R. and Tham, M. T. (2004). Performance Envelopes of Process Intensified Systems. Proceedings of International Symposium on Advanced Control of Chemical Processes. Hong Kong.

Adrian, T., Schoenmakers, H. and Boll, M. (2004). "Model predictive control of integrated unit operations: Control of a divided wall column," Chemical Engineering and Processing, 43(3), 347- 355.

Al-Arfaj, M.A.,Luyben, W.L. (2000). “Comparison of alternative control structures for an ideal two-product reactive distillation column." Industrial and Engineering Chemistry Research, 39(9), 3298- 3307.

Al-Arfaj, M.A., Luyben, W.L. (2002a). "C ontrol of ethylene glycol reactive distillation column," A.I.Ch.E. J ournal, 48(4), 905- 908.

Al-Arfaj, M. A., Luyben, W. L. (2002b). "Design and control of an olefin metathesis reactive distillation column." Chemical Engineering Science, 57(5), 715- 733.

Bisowarno, B. H., Tian, Y. C., Tade, M. 0. (2003). "Model gain scheduling control of an ethyl tert-butyl ether reactive distillation column." Industrial and Engineering Chemistry Research, 42(15), 3584- 3591.

Curry, J. E., Jackson, S. M., Stohrer, B. and van der Veen A. P. (1988). "Free radical degradation of polypropylene," Chemical Engineering Progress, 84(11), 43-46.

Keller, G. E. and Bryan, P. F. (2000). "Process engineering: Moving in new directions." Chemical Engineering Process, 96(1), 4149.

McG reavy, C. (1983). "On-line control system for chemical reaction processes. "Computers and Chemical Engieering, 7(4), 529-566.
Olanrewaju, M. J.,and Al-Arfaj M. A. (2006). "Estimator-based control of reactive distillation system: Application of an extended Kalman filtering." Chemical Engineering Science, 61(10), 3386-3399.

Pabedinskas, A., Cluett, W. R., and Balke, S. T. (1989). "Process control for polypropylene degradation during reactive extrusion." Polymer Engineering and Science, 29(15), 993-1003.

Pabedinskas, A., and Cluett, W. R. (1994). "C ontroller design and performance analysis for a reactive extrusion process" Polymer Engineering and Science, 34(7), 585-597.

Palusinski, A. O., Vrudhula, S., Znamirowski, L., Humbert, D. (2001)." Process control for microreactors." Measurement and Control 60-66.

Rogge, T., Rummler, Z., Schomburg, W. K. (2004). "Polymer micro valve with a hydraulic piezo-drive fabricated by the AMANDA process," Sensors and Actuators A 110, 206- 212.

Stankiewicz, A. (2004). Re-engineering the chemical process plant. Marcel Dekker, Inc., New York. Basel

Toledo, E. C. V., Martini, R. F., Maciel, M. R. W., Filho, R. M. (2005). "Process intensification for high operational performance target: Autorefrigerated CSTR polymerization reactor." Computers and Chemical Engineering, 29(6), 1447- 1455.

Wille, Ch., Gabski, H. P., Haller, Th., Kim, H., Unverdorben, L., and Winter, R. (2004) "Synthesis of pigments in a three-stage microreactor pilot plant-An experimental technical report," Chemical Engineering J ournal, 101, 179- 185. 